\title{
One-Step Synthesis of PEGylated Gold Nanoparticles with Tunable Surface Charge
}

\author{
Rares Stiufiuc, ${ }^{1}$ Cristian Iacovita, ${ }^{1}$ Raul Nicoara, ${ }^{1}$ Gabriela Stiufiuc, ${ }^{2}$ Adrian Florea, ${ }^{3}$ \\ Marcela Achim, ${ }^{4}$ and Constantin M. Lucaciu ${ }^{1}$ \\ ${ }^{1}$ Department of Pharmaceutical-Biophysics, Faculty of Pharmacy, "Iuliu Hatieganu" University of Medicine and Pharmacy, \\ Pasteur 6, 400349 Cluj-Napoca, Romania \\ ${ }^{2}$ Department of Solid State Physics and Advanced Technologies, Faculty of Physics, "Babes-Bolyai” University, Kogalniceanu 1, \\ 400084 Cluj-Napoca, Romania \\ ${ }^{3}$ Department of Cell and Molecular Biology, Faculty of Medicine, "Iuliu Hatieganu" University of Medicine and Pharmacy, \\ Pasteur 6, 400349 Cluj-Napoca, Romania \\ ${ }^{4}$ Department of Pharmaceutical Technology and Biopharmaceutics, Faculty of Pharmacy, "Iuliu Hatieganu" University of Medicine \\ and Pharmacy, Creanga 12, 400010 Cluj-Napoca, Romania
}

Correspondence should be addressed to Rares Stiufiuc; rares.stiufiuc@umfcluj.ro

Received 18 June 2013; Accepted 7 September 2013

Academic Editor: Donglu Shi

Copyright (C) 2013 Rares Stiufiuc et al. This is an open access article distributed under the Creative Commons Attribution License, which permits unrestricted use, distribution, and reproduction in any medium, provided the original work is properly cited.

The present work reports a rapid, simple and efficient one-step synthesis and detailed characterisation of stable aqueous colloids of gold nanoparticles (AuNPs) coated with unmodified poly(ethylene)glycol (PEG) molecules of different molecular weights and surface charges. By mixing and heating aqueous solutions of PEG with variable molecular chain and gold(III) chloride hydrate $\left(\mathrm{HAuCl}_{4}\right)$ in the presence of $\mathrm{NaOH}$, we have successfully produced uniform colloidal $5 \mathrm{~nm}$ PEG coated AuNPs of spherical shape with tunable surface charge and an average diameter of $30 \mathrm{~nm}$ within a few minutes. It has been found out that PEGylated AuNPs provide optical enhancement of the characteristic vibrational bands of PEG molecules attached to the gold surface when they are excited with both visible $(532 \mathrm{~nm})$ and NIR $(785 \mathrm{~nm})$ laser lines. The surface enhanced Raman scattering (SERS) signal does not depend on the length of the PEG molecular chain enveloping the AuNPs, and the stability of the colloid is not affected by the addition of concentrated salt solution $(0.1 \mathrm{M} \mathrm{NaCl})$, thus suggesting their potential use for in vitro and in vivo applications. Moreover, by gradually changing the chain length of the biopolymer, we were able to control nanoparticles' surface charge from -28 to $-2 \mathrm{mV}$, without any modification of the Raman enhancement properties and of the colloidal stability.

\section{Introduction}

The ability of tuning the physicochemical properties of different classes of metallic and/or semiconductor nanoparticles by controlling their size in the nanoscale put them in the limelight of the modern biomedical research. Over the past years, several classes of nanoparticles: quantum dots, polymer nanoparticles [1], dendrimers [2], liposomes [3], nanotubes [4], and nanorods [5], have been synthesized and characterized. Owing to their unique properties (enhancement of Raman signal, size-tunable surface plasmon resonance, ease of preparation and of surface functionalization), gold nanoparticles (AuNPs) have become one of the most interesting candidates for modern biomedical applications such as biosensing, targeted drug delivery and photothermal therapy $[6,7]$. So far several methods have been developed for the synthesis of AuNPs. The first developed method, also known as the citrate reduction method, has been proposed by Turkevich et al. in 1951 [8]. It allows the synthesis of almost monodisperse spherical gold nanoparticles, ranging in size from 10 to $50 \mathrm{~nm}$. However this method has two major drawbacks: it cannot be used for the preparation of smaller nanoparticles and the surface chemistry of the nanoparticles is poorly defined. The two-phase synthesis 
TABLE 1: Absorption maximum, fwhm, pH, and Zeta potential values of colloidal AuNPs reduced with different molecular weight PEGs.

\begin{tabular}{lcccc}
\hline Nanoparticles & Absorption maximum $(\mathrm{nm})$ & Fwhm $(\mathrm{nm})$ & $\mathrm{pH}$ & Zeta potential $(\mathrm{mV})$ \\
\hline AuNPs-PEG200 & 520 & 50 & 7,440 & $-28,2$ \\
AuNPs-PEG400 & 533 & 51 & 7,692 & $-25,5$ \\
AuNPs-PEG1500 & 523 & 59 & 7,485 & $-16,2$ \\
AuNPs-PEG4000 & 522 & 54 & 7,795 & $-12,1$ \\
AuNPs-PEG6000 & 519 & 49 & 7,556 & $-7,72$ \\
AuNPs-PEG10000 & 517 & 47 & 7,714 & $-2,16$ \\
AuNPs-PEG20000 & 524 & 49 & 7,552 & $-1,94$ \\
\hline
\end{tabular}

developed by Brust et al. more than 40 years ago allowed the synthesis of smaller gold nanoparticles and their subsequent functionalization with thiolated molecules [9]. The success of this method is mainly due to the possibility of incorporating chemical functionalities on the nanoparticle surface as novel biomedical applications require nanoparticles functionalized with different species of biomolecules.

As a consequence, there is a strong demand for developing synthesis strategies capable of producing ready to use biocompatible nanoparticles in a facile, rapid, and cost friendly manner. On the other hand, polyethylene glycol (PEG) is nowadays one of the most used biopolimers, being a principal component in different classes of therapeutic agents which are already in clinical use. It is inexpensive, versatile, and FDA approved for clinical use in USA [10]. Two major advantages can be highlighted in the case of PEGylated nanoparticles with respect to the nonfunctionalized ones: a considerable increase in the circulation time and a much better hydrophilicity. The synthesis methods of PEGylated AuNPs reported in the literature so far are generally carried out in a two-step process involving the formation of spherical AuNPs (by employing toxic chemical reducing agents) followed by a subsequent addition of thiol modified PEG molecules [6].

We report herein a very simple, biocompatible, cost effective and rapid synthesis method of PEGylated AuNPs by using unmodified PEG which has a dual function, as a reducing and stabilizing agent. All the synthesis reactions were carried out in very mild conditions. By employing PEG of different chain lengths, we were able to tune the surface charge of the nanoparticles in an interval ranging between -28 and $-2 \mathrm{mV}$ in a very controllable and reproducible manner. It has turned out that the creation of an alkaline environment by using $\mathrm{NaOH}$ in the preparation method is an essential condition for the formation of AuNPs in a very rapid manner (few seconds) [11]. The as-prepared colloids have been investigated by UV-VIS absorption spectroscopy, transmission electron microscopy (TEM), zeta potential measurements, and surface-enhanced Raman spectroscopy.

\section{Materials and Methods}

2.1. Chemicals. Gold(III) chloride hydrate was purchased from Sigma-Aldrich (Germany). PEGs ( $\mathrm{HO}-\left[\mathrm{CH}_{2}-\mathrm{CH}_{2}-\right.$ $\left.\mathrm{O}]_{n}-\mathrm{H}\right)$ with different molecular weights $(\mathrm{M}=200,400,1500$, $4000,6000,10000$, and $20000 \mathrm{~kg} / \mathrm{mol}$, hereafter denoted
PEG200, 400, 1500, 4000, 6000, 10000, and 20000) were obtained from Carl Roth (Germany) and used without further purification. Sodium hydroxide was provided by VWR International. Double distilled water (18.2 M $\Omega$, Barnstead EASYPure ROdi) was used as solvent.

2.2. Synthesis of Gold Colloids. The preparation of gold nanoparticles was performed by the addition of an aqueous $\mathrm{HAuCl}_{4}$ solution to an aqueous PEG solution (of different molecular weights) containing $\mathrm{NaOH}$. In a typical procedure, $340 \mu \mathrm{L}$ of PEG200 and $0.75 \mathrm{~mL}$ of $\mathrm{NaOH} 1 \%$ were added to $45 \mathrm{~mL}$ of water in an Erlenmeyer glass and heated to $50^{\circ} \mathrm{C}$ on a magnetic stirrer with heating option. A $5 \mathrm{~mL}$ aqueous solution containing $19.7 \mathrm{mg} \mathrm{HAuCl}_{4}$ was then added rapidly under vigorous stirring. The resulting solution was then slowly heated up to $80^{\circ} \mathrm{C}$, where the transparent solution converted immediately to the characteristic ruby red color, which indicated the formation of PEG coated gold nanoparticles. A similar preparation method has been applied for the synthesis of gold nanoparticles with different molecular weight PEGs (i.e., 400, 1500, 4000, 6000, 10000, and 20000), keeping constant the amount of $\mathrm{PEG}, \mathrm{NaOH}$, and $\mathrm{HAuCl}_{4}$. Since the PEG with molecular weight between 1500 and 20000 are found in solid form, $6 \mathrm{~g}$ of each was used in the preparation method. The $\mathrm{pH}$ values of all PEG reduced gold colloids right after the preparation were around 7.5 (Table 1). The gold nanoparticles biofunctionalized with PEG of different molecular weights are stable for months at room temperature without any change of their properties, indicating that the PEG is an efficient stabilizer for gold nanoparticles.

2.3. Nanoparticle Characterization. The prepared colloidal PEG-AuNPs were characterized by using ultraviolet-visible spectroscopy (UV-VIS), transmission electron microscopy (TEM), zeta potential measurements, and surface enhanced raman spectroscopy (SERS).

The UV-VIS spectra of the synthesized colloidal PEGAuNPs were recorded on an UV-VIS-NIR diode array spectrometer (ABL\&E Jasco, Japan), using standard quartz cells at room temperature, over a spectral range between $400 \mathrm{~nm}$ and $1000 \mathrm{~nm}$ and a spectral resolution of $2 \mathrm{~nm}$. For TEM examination, $5 \mu \mathrm{L}$ drops of aqueous suspensions containing PEG coated AuNPs were deposited on carbon-coated copper grids. After 15 minutes, the excess water was removed by the filter paper; then, the samples were left to dry under the ambient air. TEM images were taken on a Jeol JEM 
1010 transmission electron microscope (JEOL Ltd., Tokyo, Japan), equipped with a Mega VIEW III camera (Olympus, Soft Imaging System, Münster, Germany), operating at $80 \mathrm{kV}$. The Zeta potential measurements were carried out by using a Zetasizer Nano-ZS (Malvern Instruments, Worcestershire, UK). Each sample was measured three times consecutively. The $\mathrm{pH}$ measurements were done by using an inoLab $7310 \mathrm{pH}$ meter (Wissenschaftlich-Technische Werkstätten $\mathrm{GmbH}$, Weilheim, Germany). Raman and SERS spectra were recorded using Advantage 532 and Advantage 785 Raman spectrometers (Delta Nu Inc., Laramie, WY) equipped with a doubled frequency Nd:YAG laser emitting at $532 \mathrm{~nm}(100 \mathrm{~mW}$ laser power) and a HeNe laser emitting at $785 \mathrm{~nm}(60 \mathrm{~mW}$ laser power), respectively. The spectral resolution of the two spectrometers was $5 \mathrm{~cm}^{-1}$. All the Raman and the SERS spectra were recorded in $1 \mathrm{~mL}$ glass vials filled with $700 \mu \mathrm{L}$ analyte solution. An accumulation time of $30 \mathrm{~s}$ was used, the final spectrum being the average of previous 5 recordings. The calculated molar concentration of PEG-coated AuNPs in the final solution was $1.2 \times 10^{-6} \mathrm{M}$ [12].

\section{Results and Discussion}

3.1. UV-VIS Spectroscopy and TEM. The morphology of all the produced gold colloids has been characterized by using UV-VIS spectroscopy and TEM. The absorption maximum of the measured UV-VIS spectra of the colloidal solution gives information on the average particle size, whereas their full width at half-maximum (fwhm) can be used to estimate particle dispersion [13]. The UV-VIS spectra of the all prepared colloidal AuNPs (Figure 1, curves from A to G) exhibits a narrow and well-defined absorption band having the absorption maximum around $520 \mathrm{~nm}$ and a fwhm of about $50 \mathrm{~nm}$ (Table 1), indicating a narrow size and shape distribution of the AuNPs immediately after synthesis [14]. The values of the absorption maximum are a characteristic for surface plasmon resonance (SPR) absorbance of the spherical AuNPs induced by the interaction between the incoming light and the conduction electrons of the spherical metallic nanoparticle [15]. The blue/red shift of the SPR maximum is a consequence of a decrease/increase in the refractive index surrounding the AuNPs due to the modification of PEG molecular chain length in the preparation method [16]. The values of SPR maximum and their corresponding fwhm values did not change during several months, thus suggesting the high stability of the gold colloidal solutions. Moreover, the slight variation of these values indicates that spherical AuNPs coated with PEG of different molecular weight and with approximately similar particles size and dispersion could be reproducibly obtained.

The TEM characterization of all prepared colloidal gold solutions supports the above experimental observations. Representative TEM images of colloidal gold nanoparticles coated with PEG200 and PEG4000 are given in Figure 2. In both cases the gold nanoparticles are predominantly spherical in shape, with a diameter between $10 \mathrm{~nm}$ and $80 \mathrm{~nm}$. Moreover, by comparing the higher magnification TEM images taken in the case of two classes of PEGylated

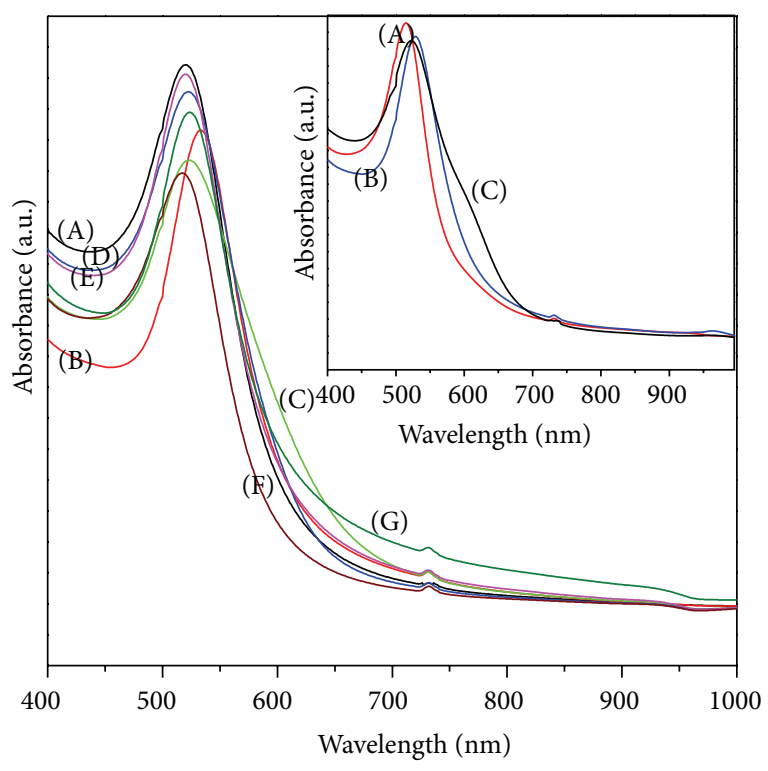

FIGURE 1: UV-VIS spectroscopy: UV-VIS spectra of AuNPs coated with PEG200 (A), PEG400 (B), PEG1500 (C), PEG4000 (D), PEG6000 (E), PEG10000 (F), and PEG20000 (G). The inset displays the UV-VIS spectra of PEG4000-AuNPs obtained (A) by adding the $\mathrm{NaOH}$ to an aqueous solution of PEG4000 and $\mathrm{HAuCl}_{4}$, (B) by adding the PEG4000 to an aqueous solution of $\mathrm{HAuCl}_{4}$ and $\mathrm{NaOH}$, and (C) by using half amount of PEG4000 in the preparation method. The single peak in all spectra indicates the successful formation of spherical PEGylated AuNPs. The order of $\mathrm{NaOH}$ addition in the preparation method does not influence the formation of spherical gold nanoparticles, while the amount of PEG does.

gold nanoparticles (AuPEG200 and AuPEG4000), it can be easily observed that most of the gold nanoparticles coated with either PEG200 or PEG4000 have similar sizes (Figures 2 (b) and 2(c)). The average diameter in each case is about $30 \mathrm{~nm}$. The $5 \mathrm{~nm}$ PEG layer, uniformly surrounding the gold core of a single nanoparticle and visible in the inset of the Figure 2(a), was included in the nanoparticles' size estimation. A closer inspection of the TEM images taken on AuNPs coated with PEG200 shows up single PEGylated AuNPs, dimers, trimers (inset of Figure 2(a)) or small clusters apart from bigger random agglomerations. The AuNPs coated with PEG4000 randomly self-assemble in agglomerations as well (Figures 2(c) and 2(d)). Besides this, we have observed row of AuNPs in a few TEM images (inset of Figure 2(c)). We believe that the aggregation of PEGylated AuNPs in different patterns is induced by their adsorption on the surface. Upon water evaporation, the PEGylated AuNPs were adsorbed on the substrate and self-assembled through hydrogen bonds formed between PEG molecules. This is not the case in the solution, where the PEG molecules bounded to gold nanoparticles usually form hydrogen bonds with the water molecules increasing the hydrophilicity of these nanoparticles and preventing their agglomeration through steric repulsion. In addition, the PEGylated AuNPs possess a negative charge as confirmed by Zeta potential measurements (Table 1). The value of the negative surface charge gradually 


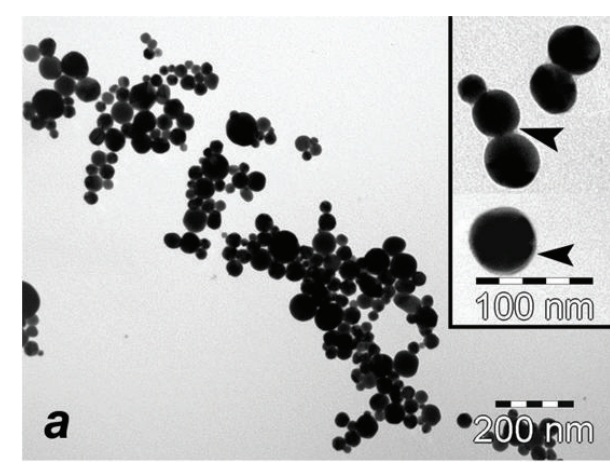

(a)

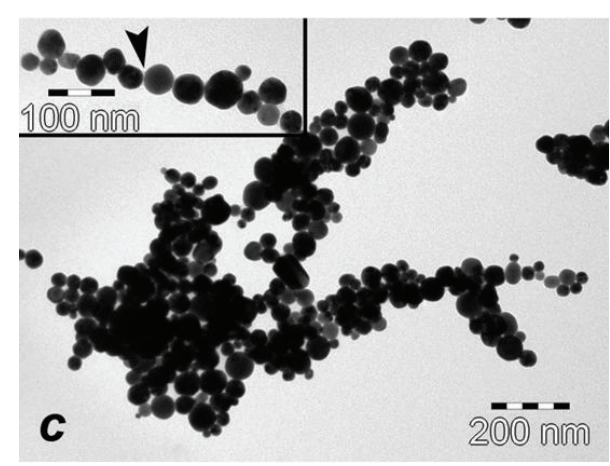

(c)

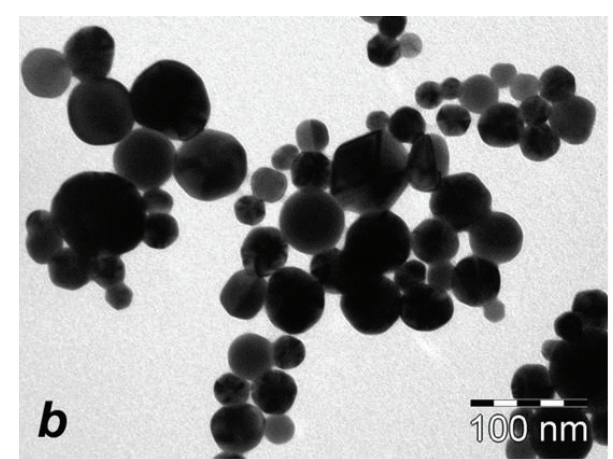

(b)

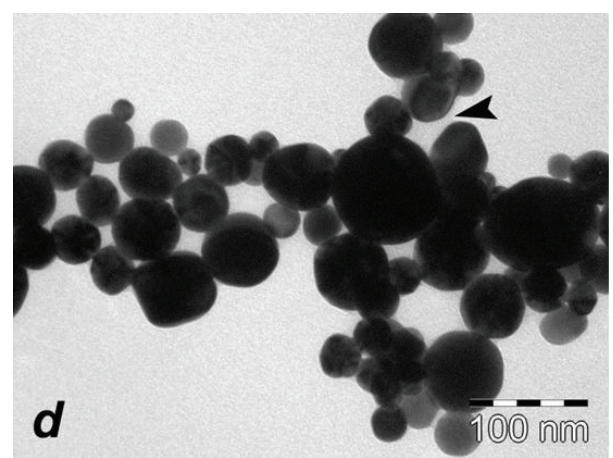

(d)

FIGURE 2: TEM characterization: TEM images at different magnification of AuNPs reduced with either PEG200 ((a) and (b)) or PEG4000 ((c) and (d)). The inset of (a) displays a single gold nanoparticle, a dimer and a trimer coated with a $5 \mathrm{~nm}$ PEG larger. The inset of (c) shows PEG4000-AuNPs self-assembled in a row. Spherical AuNPs coated with PEG200 and 4000 with similar narrow size distribution are visible.

TABLE 2: The $\mathrm{pH}$ of the colloidal solution as a function of additional $\mathrm{NaOH}$ volume.

\begin{tabular}{lccccccccccccc}
\hline $\mathrm{NaOH}(\mathrm{mL})$ & 0 & 0,1 & 0,2 & 0,3 & 0,4 & 0,45 & 0,5 & 0,55 & 0,6 & 0,65 & 0,7 & 0,75 & 0,8 \\
\hline $\mathrm{pH}$ & 3,383 & 3,519 & 3,84 & 4,179 & 4,605 & 4,92 & 5,048 & 5,359 & 6,566 & 7,127 & 7,715 & 7,894 & 9,54 \\
\hline
\end{tabular}

decreases by increasing the PEG chain length used in the preparation method. A surface charge value of $-28.2 \mathrm{mV}$ in the case of AuNPs coated with short-chain PEGs (PEG200) is a strong proof that the main mechanism responsible for colloidal stability is the electrostatic repulsion. Upon increasing the PEG chain length, the negative charge drops up to a minimum of $-2 \mathrm{mV}$ (for PEG20000-AuNPs), diminishing the electrostatic repulsion between the PEGylated nanoparticles. However, the stability of almost neutral AuNPs coated with long-chain PEG is now assured by the steric repulsion between the PEG chains that increases along with increasing PEG chain length. The very low value of nanoparticles surface charge could represent a major advantage in the case of their potential use in the biomedical applications since this class of PEGylated nanoparticles could strongly reduce their association with nontargeted serum and tissue proteins [10].

3.2. The Role of $\mathrm{NaOH}$. $\mathrm{NaOH}$ represents an important factor in the formation of the AuNPs since it generates -OH groups in the aqueous solution, shifting the solution $\mathrm{pH}$ towards an alkaline environment that promotes the very fast reduction of the $\mathrm{Au}^{3+}$ ions to $\mathrm{Au}^{0}$ atoms. Table 2 summarizes the evolution of the $\mathrm{pH}$ value of an aqueous solution of PEG4000- $\mathrm{HAuCl}_{4}$ at $80^{\circ} \mathrm{C}$, as a function of the amount of $\mathrm{NaOH}$ added to the solution. As expected, the solution $\mathrm{pH}$ gradually increases as the amount of $\mathrm{NaOH}$ in the solution is increased. For a very low amount of $\mathrm{NaOH}$, the color of the solution is transparent, changing to light blue for $0.3 \mathrm{~mL}$ of $\mathrm{NaOH}(1 \%)$. Upon further addition of $\mathrm{NaOH}$, the light blue converts to dark blue, dark purple, and for $0.65 \mathrm{~mL}$ of $\mathrm{NaOH}$ (solution $\mathrm{pH}$ of 7.127), the color becomes ruby red, indicating the formation of the spherical AuNPs. At this point the ruby red color persists as the amount of $\mathrm{NaOH}$ in the solution is further increased. The shift of the solution $\mathrm{pH}$ to higher values accelerates the decomposition of $\mathrm{AuCl}_{3}$ and consequently the reduction of gold ions by PEG. Most probably, this occurs through the oxidation of the hydroxyl end groups to aldehyde groups, as it has already been proposed for PEGylated silver nanoparticles $[17,18]$. The absence of $\mathrm{NaOH}$ from the PEG-HAuCl $\mathrm{P}_{4}$ aqueous solution makes impossible the formation of PEGylated spherical AuNPs in a very short time. It might be possible that, under vigorous stirring at elevated temperatures, the formation of PEGylated spherical 
AuNPs could be accomplished in a very long period of time as it was the case for silver nanoparticles $[16,19,20]$. Such a synthesis method is beyond the purpose of the current study.

In order to strengthen the major role of $\mathrm{NaOH}$ in accelerating the formation of PEGylated AuNPs, we have added the $\mathrm{NaOH}$ solution at different stages in the preparation method. The rapid addition of $\mathrm{NaOH}$ on the aqueous solution containing PEG4000 and $\mathrm{HAuCl}_{4}$ heated at $80^{\circ} \mathrm{C}$ leads to the formation of stable PEGylated AuNPs (inset of Figure 1, curve A). When an aqueous solution of PEG4000 is added to an aqueous solution containing $\mathrm{HAuCl}_{4}$ and $\mathrm{NaOH}$ at $50^{\circ} \mathrm{C}$ and further heated at $80^{\circ} \mathrm{C}$, the formation of spherical AuNPs does not proceed immediately. During 10 minutes at boiling conditions the transparent solution converted slowly into the characteristic ruby red color, indicating the formation of spherical PEG coated AuNPs (inset of Figure 1, curve B). Therefore, the order in which the $\mathrm{NaOH}$ is introduced in the preparation method seems to have a minor impact on the formation of PEGylated AuNPs.

3.3. The Role of PEG. The formation of AuNPs was also investigated by changing the amount of PEG in the preparation method. By reducing to half the initial amount of PEG4000 (6g to $3 \mathrm{~g}$ ), a shoulder at $610 \mathrm{~nm}$ appears in the UV-VIS spectrum (inset of Figure 1, curve C) near the SPR band of spherical AuNPs. This suggests the existence in the colloidal solution of AuNPs with a different shape. The use of only $1 \mathrm{~g}$ of PEG4000 in the preparation method induces the formation of AuNPs, but these nanoparticles are not stable aggregating in a very short period. Therefore, the amount of PEG plays an important role in both formation and stabilization of spherical AuNPs. Moreover, $1 \mathrm{~g}$ of PEG4000 yields an $\mathrm{OH} / \mathrm{Au}^{3+}$ molar ratio of 5 , a value which is very close to that obtained by using $6 \mathrm{~g}$ of PEG20000, which produces well-defined spherical AuNPs (Figure 1 curve G). It turns out that the PEG chain length also contributes to the stabilization of spherical AuNPs.

3.4. SERS Measurements. The promising biomedical applications of PEGylated spherical AuNPs as Raman tags require an assessment of their SERS properties. Normally, molecules with strong Raman signals that bound to the gold nanoparticles are used to probe the SERS activity of the as-prepared gold colloids [21]. This was not our case, since the SERS spectra were directly acquired on the aqueous solution of PEG reduced AuNPs by using both 532 and 785 laser lines (Figures 3 and 4). The Raman spectrum of pure PEG200 was chosen as a reference for the characterization of SERS activity of the PEG-reduced gold colloids. The Raman spectrum is dominated by strong phonon bands over all the spectral range (Figures 3 and 4), and their assignment can be found in the literature [22]. As it can be observed in Figure 3, all the characteristic vibrational bands of PEG can be identified in the SERS spectra acquired with the $532 \mathrm{~nm}$ laser line. For this excitation wavelength, the AuNPs adsorb resonantly the maximum light and consequently enhance the vibrational bands of the PEG molecules adsorbed onto the nanoparticles surface. A slightly different situation has been observed in

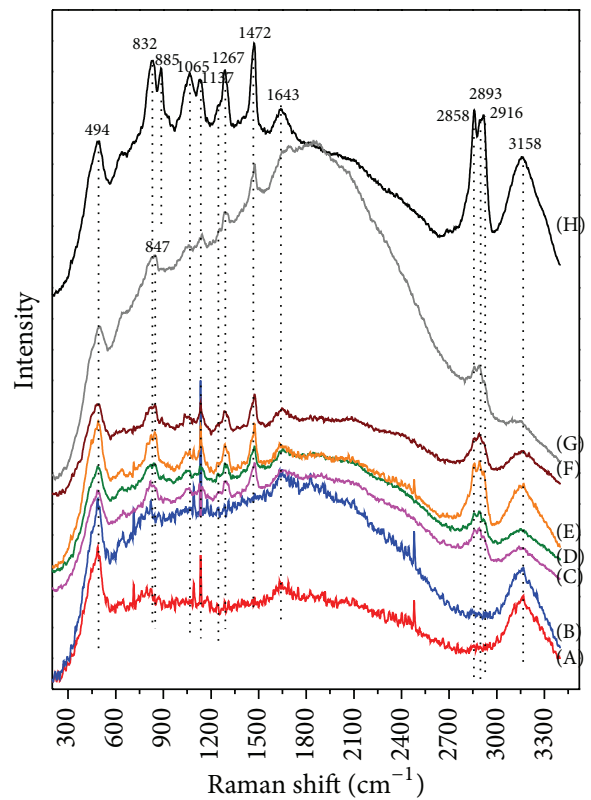

FIGURE 3: SERS analysis: SERS spectra (employing the $532 \mathrm{~nm}$ laser line) of AuNPs coated with PEG200 (A), PEG400 (B), PEG1500 (C), PEG4000 (D), PEG6000 (E), PEG10000 (F) and PEG20000 (G). Raman spectrum of the pure PEG200 (H). The spectra were shifted for clarity. All the specific vibrational bands of PEG molecules attached to the gold nanoparticles are clearly visible. The SERS signal is sensitive to PEG molecular chain length.

the case of AuNPs coated with PEG200 and 400, where the enhancement has been observed only for three vibrational bands $\left(494 \mathrm{~cm}^{-1}, 1643 \mathrm{~cm}^{-1}\right.$, and $\left.3158 \mathrm{~cm}^{-1}\right)$. This might indicate a different adsorption geometrical conformation of PEG200 and 400 at the gold nanoparticle surface with respect to the rest of PEG polymers employed in the synthesis [10]. It was shown that depending on the density of PEG molecules covering the gold surface they can adopt two different geometrical arrangements [10]: high molecular PEG density (PEG200 and 400) promotes a brush type arrangementthe PEG molecules are perpendicular to the gold surfacewhile a low molecular PEG density (PEG 1500 to 20000) gives rise to a mushroom conformation-the PEG molecules lie parallel the gold surface. All the vibrational bands of PEG are missed in the SERS spectra of these nanoparticles excited with 785 laser line. Instead, the SERS spectra acquired on colloidal AuNPs coated with PEG1500 to 20000 exhibit characteristic vibrational bands of PEG. The bands between $800 \mathrm{~cm}^{-1}$ and $900 \mathrm{~cm}^{-1}$ (skeleton vibration of PEG) as well as the band at $1138 \mathrm{~cm}^{-1}$ (twisting vibration of $\mathrm{CH}_{2}$ ) dominate over the entire spectral range, while other vibrational bands of PEG appear faintly in the SERS spectra. This could be explained by the fact that, at NIR excitation wavelength, the system is not anymore in a surface enhanced resonant Raman spectroscopy (SERRS) regime (as it was the case when employing the $532 \mathrm{~nm}$ excitation laser line) but in a simple SERS one. However, the importance of using this excitation laser in the biomedical applications resides in the fact that two major components of human body (hemoglobin 


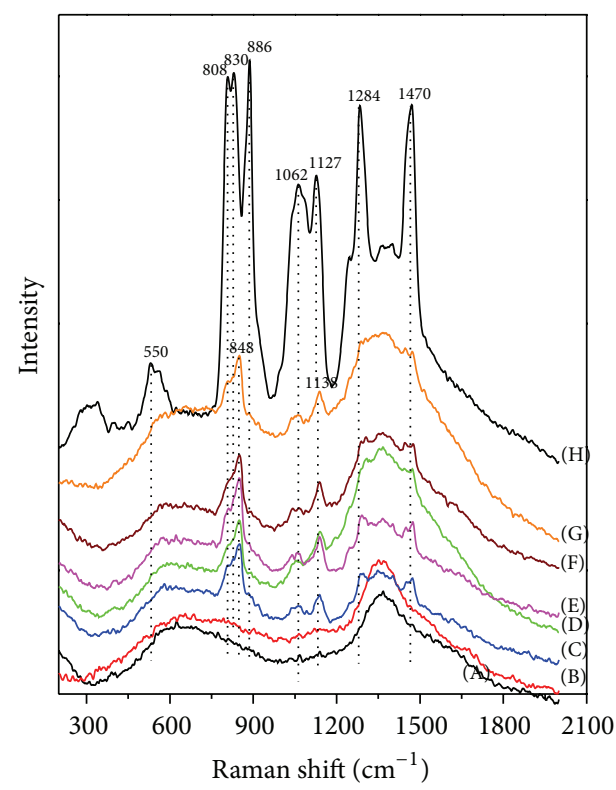

FIGURE 4: SERS analysis: SERS spectra (employing the $785 \mathrm{~nm}$ laser line) of AuNPs coated with PEG200 (A), PEG400 (B), PEG1500 (C), PEG4000 (D), PEG6000 (E), PEG10000 (F) and PEG20000 (G). Raman spectrum of the pure PEG200 (H). The spectra were shifted for clarity. Some specific vibrational bands of PEG molecules attached to the gold nanoparticles are clearly visible. The SERS signal is sensitive to PEG molecular chain length.

and water) have a minimal absorption at this wavelength [23]. As a further proof of their possible use as Raman tags, we have acquired the SERS spectra of all colloidal solutions upon the addition of concentrated salt solution $(\mathrm{NaCl} 0.1 \mathrm{M})$. It has been observed that all the SERS spectra do not change, meaning that the PEG layer protects the AuNPs from aggregation (not shown). These observations indicate that the as-obtained PEGylated AuNPs enable the enhancement of the characteristical vibrational bands of PEG, thus showing their potential use of these nanoparticles as Raman tags without further attachment of a reporter molecule. The achievement of a NIR Raman enhancement extends the potential applications of these nanoparticles for in vivo spectroscopic detection.

\section{Conclusions}

In this paper we have proposed a rapid, one-step and green method for the synthesis of the AuNPs biofunctionalized with unmodified PEG molecules. The UV-VIS spectra have showed that the PEG of different molecular weights in conjunction with $\mathrm{NaOH}$ has a remarkable capability to produce spherical PEGylated AuNPs at $80^{\circ} \mathrm{C}$ with a great stability in time. The alkaline environment, created by the addition of a certain amount of $\mathrm{NaOH}$ in the solution, favors the very fast formation of spherical PEGylated AuNPs. By varying the chain length of the PEG molecules used in the preparation method, we were able to finely tune the surface charge of the as-obtained spherical PEGylated AuNPs in a range between
$-28 \mathrm{mV}$ and $-2 \mathrm{mV}$. The SERS measurements (acquired with two laser lines: $532 \mathrm{~nm}$ and $785 \mathrm{~nm}$ ) have shown that all PEGylated AuNPs have a characteristic Raman spectrum, thus enabling them to be used as Raman tags without further attachment of a Raman report.

\section{Conflict of Interests}

The authors declare that they have no conflict of interests.

\section{Acknowledgment}

This research was supported by CNCSIS-UEFISCDU, Project no. PN-II-RU TE 259/2010 and Project no. PN-II-ID-PCE2011-3-0954.

\section{References}

[1] P. Xu, E. A. van Kirk, Y. Zhan, W. J. Murdoch, M. Radosz, and Y. Shen, "Targeted charge-reversal nanoparticles for nuclear drug delivery," Angewandte Chemie, vol. 46, no. 26, pp. 4999-5002, 2007.

[2] C. M. Paleos, D. Tsiourvas, Z. Sideratou, and L. Tziveleka, "Acidand salt-triggered multifunctional poly(propylene imine) dendrimer as a prospective drug delivery system," Biomacromolecules, vol. 5, no. 2, pp. 524-529, 2004.

[3] E. T. Kisak, B. Coldren, C. A. Evans, C. Boyer, and J. A. Zasadzinski, "The vesosome-a multicompartment drug delivery vehicle," Current Medicinal Chemistry, vol. 11, no. 2, pp. 199219, 2004.

[4] W. Wu, S. Wieckowski, G. Pastorin et al., "Targeted delivery of amphotericin B to cells by using functionalized carbon nanotubes," Angewandte Chemie, vol. 44, no. 39, pp. 6358-6362, 2005.

[5] A. K. Salem, P. C. Searson, and K. W. Leong, "Multifunctional nanorods for gene delivery," Nature Materials, vol. 2, no. 10, pp. 668-671, 2003

[6] P. Ghosh, G. Han, M. De, C. K. Kim, and V. M. Rotello, "Gold nanoparticles in delivery applications," Advanced Drug Delivery Reviews, vol. 60, no. 11, pp. 1307-1315, 2008.

[7] L. Dykman and N. Khlebtsov, "Gold nanoparticles in biomedical applications: recent advances and perspectives," Chemical Society Reviews, vol. 41, no. 6, pp. 2256-2282, 2012.

[8] J. Turkevich, P. C. Stevenson, and J. Hillier, "A study of the nucleation and growth processes in the synthesis of colloidal gold," Discussions of the Faraday Society, vol. 11, pp. 55-75, 1951.

[9] M. Brust, M. Walker, D. Bethell, D. J. Schiffrin, and R. Whyman, "Synthesis of thiol-derivatised gold nanoparticles in a twophase liquid-liquid system," Journal of the Chemical Society, Chemical Communications, no. 7, pp. 801-802, 1994.

[10] J. V. Jokerst, T. Lobovkina, R. N. Zare, and S. S. Gambhir, "Nanoparticle PEGylation for imaging and therapy," Nanomedicine, vol. 6, no. 4, pp. 715-728, 2011.

[11] R. Stiufiuc, C. Iacovita, C. M. Lucaciu et al., "SERS-active silver colloids prepared by reduction of silver nitrate with short-chain polyethylene glycol," Nanoscale Research Letters, vol. 8, article 47, 5 pages, 2013.

[12] X. Liu, M. Atwater, J. Wang, and Q. Huo, "Extinction coefficient of gold nanoparticles with different sizes and different capping ligands," Colloids and Surfaces B, vol. 58, no. 1, pp. 3-7, 2007. 
[13] G. Mie, "Beiträge zur Optik trüber Medien, speziell kolloidaler Metallösungen," Annals of Physics, vol. 25, pp. 377-445, 1908.

[14] C. F. Bohren and D. R. Huffman, Absorption and Scattering of Light by Small Particles, John Wiley \& Sons, New York, NY, USA, 1998.

[15] D. A. Handley, Methods for Synthesis of Colloidal Gold: Colloidal Gold Principles, Methods, and Applications, Academic Press, San Diego, Calif, USA, 1998.

[16] S. Nam, D. V. Parikh, B. D. Condon, Q. Zhao, and M. YoshiokaTarver, "Importance of poly(ethylene glycol) conformation for the synthesis of silver nanoparticles in aqueous solution," Journal of Nanoparticle Research, vol. 13, no. 9, pp. 3755-3764, 2011.

[17] L. M. Liz-Marzán and I. Lado-Touriño, "Reduction and stabilization of silver nanoparticles in ethanol by nonionic surfactants," Langmuir, vol. 12, no. 15, pp. 3585-3589, 1996.

[18] M. Andersson, V. Alfredsson, P. Kjellin, and A. E. C. Palmqvist, "Macroscopic alignment of silver nanoaparticles in revers hexagonal liquid crystalline templates," Nano Letters, vol. 2, no. 12, pp. 1403-1407, 2002.

[19] L. Bo, W. Yang, M. Chen, J. Gao, and Q. Xue, "A simple and "green" synthesis of polymer-based silver colloids and their antibacterial properties," Chemistry and Biodiversity, vol. 6, no. 1, pp. 111-116, 2009.

[20] W. Li, Y. Guo, and P. Zhang, "SERS-active silver nanoparticles prepared by a simple and green method," Journal of Physical Chemistry C, vol. 114, no. 14, pp. 6413-6417, 2010.

[21] X. Qian, X. Peng, D. O. Ansari et al., "In vivo tumor targeting and spectroscopic detection with surface-enhanced Raman nanoparticle tags," Nature Biotechnology, vol. 26, no. 1, pp. 8390, 2008.

[22] M. Kozielskia, M. Mühle, and Z. Blaszczak, "The Raman scattering study of selected polyoxyethyleneglycols," Journal of Molecular Liquids, vol. 111, pp. 1-5, 2004.

[23] U. Mahmood and R. Weissleder, "Near-infrared optical imaging of proteases in cancer," Molecular Cancer Therapeutics, vol. 2, pp. 489-496, 2003. 

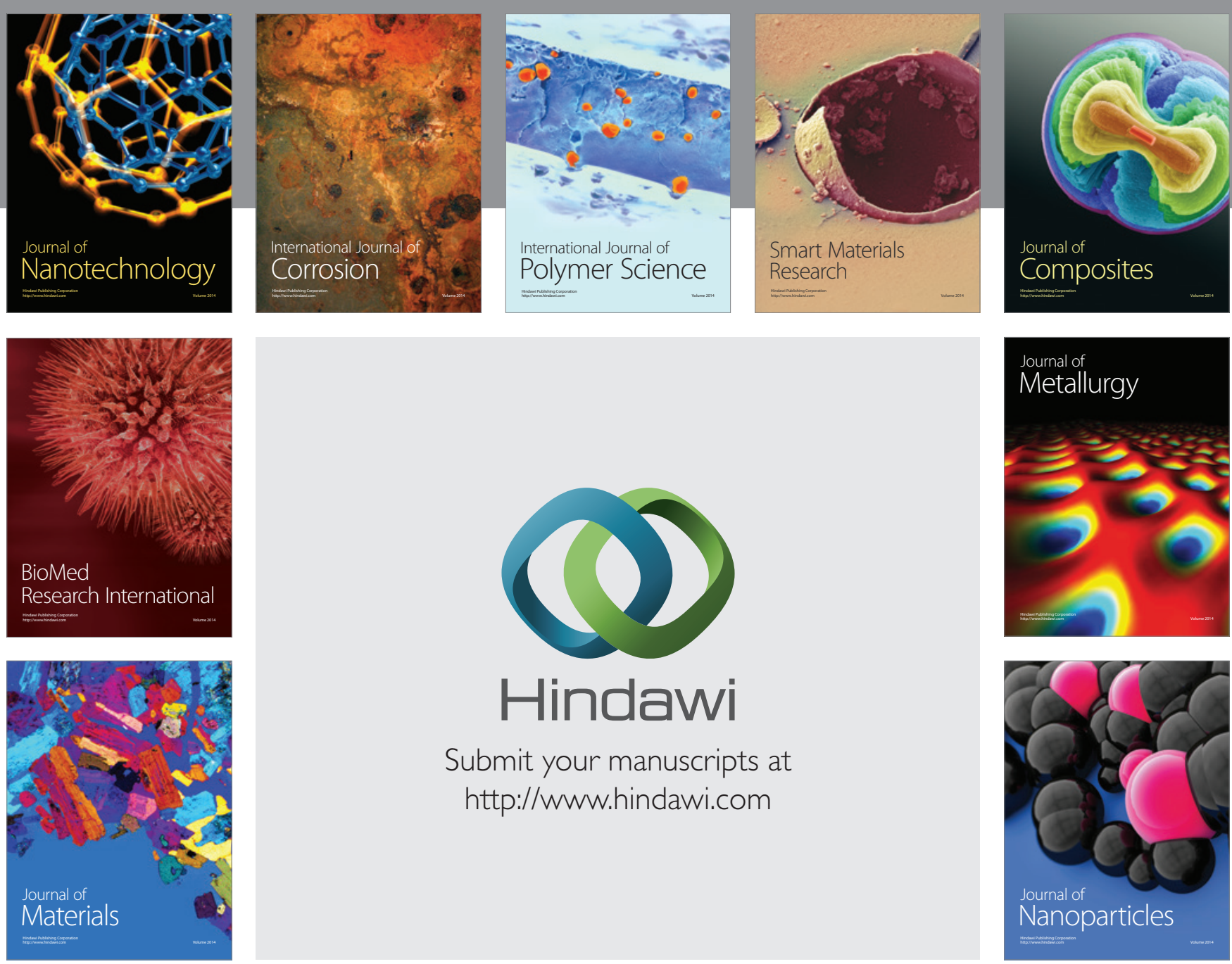

Submit your manuscripts at http://www.hindawi.com
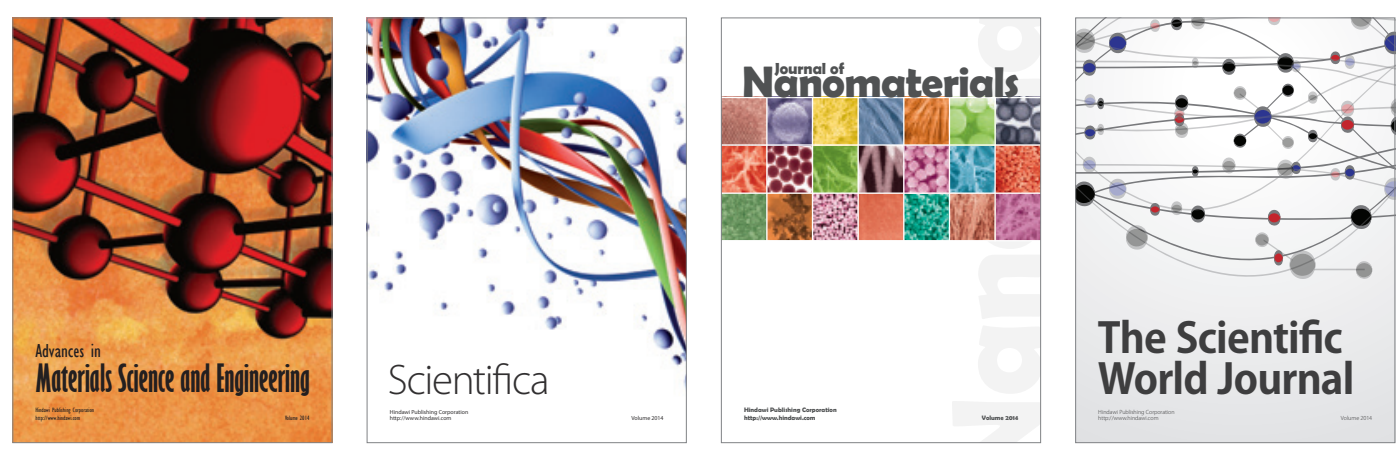

\section{The Scientific World Journal}
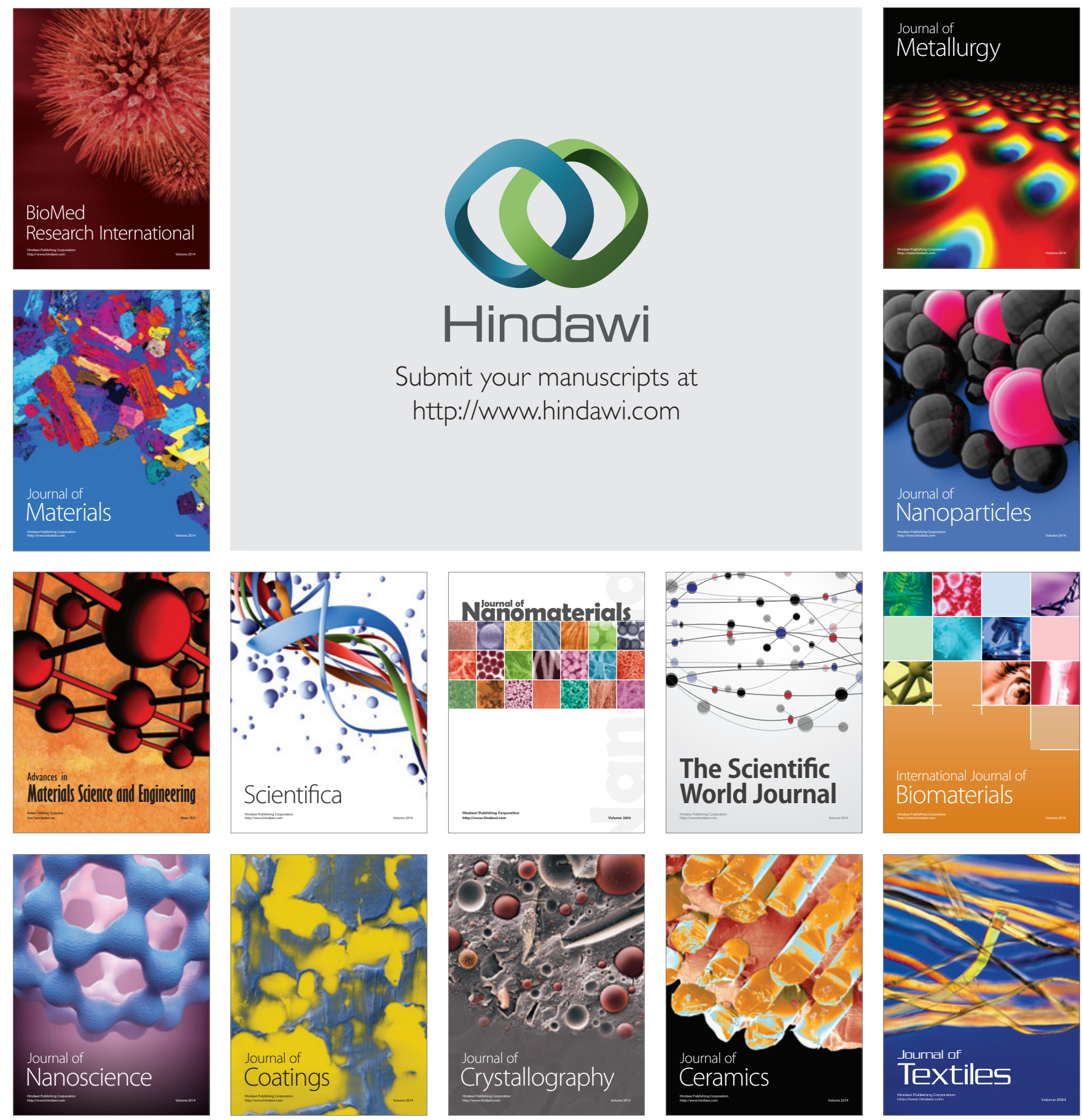